\title{
La métamorphose du célibataire chez Rachilde
}

Vicky Gauthier, Université du Québec à Chicoutimi

Des détails d'apparence insignifiante font des monstres.

Il suffit de réunir tous ces détails pour posséder le secret de la formule magique créant des féminités épouvantables.

(Rachilde, 1893)

Écrivant et publiant à une époque où la misogynie est à son paroxysme, l'auteure française Rachilde (1860-1953) semble de prime abord ne pas échapper à cette tendance : son œuvre, comme la littérature décadente, verse dans l'insolite, le morbide, la folie, la névrose, la perversion ; la femme y est considérée comme l'Autre, l'ennemie de l'homme entraînant le mal et la destruction. La présence de Rachilde au sein du cercle très fermé des auteurs décadents renforce cette impression, elle qui a su capter l'intérêt des plus rébarbatifs à l'égard des femmes écrivaines, comme Barbey d'Aurevilly et Léon Bloy. Néanmoins, on retrouve aussi chez Rachilde des personnages de femmes fortes et, dans une certaine mesure, indépendantes, libres, venant menacer l'ordre établi par leur seule présence subversive, le plus souvent à teneur fantastique.

Rappelons qu'à la fin du dix-neuvième siècle, nous assistons à un retour important du fantastique, et ce, avec un souffle de renouveau : celui du vice, de la cruauté et de la perversité tirant ses sources dans le développement des sciences humaines. Un personnage fort intéressant fait également son entrée sur la scène du roman (mais aussi de la société) : le célibataire. Dans son ouvrage Les Célibataires du fantastique (2002), Nathalie Prince montre que le célibataire devient peu à peu le héros de prédilection de ce genre de récits de la fin du dix-neuvième siècle, et expose la façon dont ce dernier entraîne l'émergence d'un fantastique plus intime, puisque son «narcissisme singulier, voire dévoyé, passe par un culte de soi et une constante analyse de soi » (15), d'où la proximité de ce type de fantastique avec le fantasme et l'hallucination. L'objet de peur fantastique fin-de-siècle se déplace de l'extérieur vers l'intérieur, de l'objet vers le sujet : la folie peut, à tout instant, surgir en l'être humain. Dès lors, ce fantastique devient, selon Prince, un fantastique de l'inadmissible, plus précisément un fantastique du monstrueux 
moral (Fantastique, 58). L'objet de peur se situe, dorénavant, dans « une raison morale dont les lois paraissent tout à la fois bafouées et perverties [par] d'inquiétantes expériences sexuelles » (58).

Pour Prince, Rachilde participe à cette tendance fantastique de la fin du dixneuvième siècle. En effet, à l'intérieur de ses romans, on retrouve fréquemment ce personnage à la Dorian Gray ou à la Des Esseintes, notamment dans Le Mordu (1889), Les Hors Nature (1897), Refaire l'Amour (1927). Mais plus encore, et c'est peut-être là sa plus grande originalité (qui mérite qu'on y porte attention), une autre figure, plus marginale et peu répandue, émerge au sein de sa fiction; le célibataire dandy et dilettante - si cher aux décadents - est, chez elle, femme. Également misanthrope désabusée - et peut-être même davantage puisqu'elle est consciente de son «pouvoir» et de ses possibilités d'action dans le monde dans lequel elle vit - la femme célibataire chez Rachilde devient une force sur-naturelle et inquiétante, dont l'esprit, le corps et les traits en sont les signes prophétiques. Cet article portera sur l'étude de deux de ses romans $L a$ Jongleuse (1900) et L'Animale (1893) et s'intéressera tout particulièrement à leurs héroïnes respectives, Éliante et Laure, qui constituent deux profils, deux prototypes du personnage de la femme célibataire. Pour ce faire, la définition du célibataire élaborée par Prince sera tout d'abord examinée, pour ensuite s'intéresser au cas de Rachilde, à savoir sa version féminine du célibataire fin-de-siècle, et ce, à travers les deux romans à l'étude, en vue d'obtenir une éventuelle définition de la femme célibataire rachildienne.

\section{Le célibataire fantastique selon Prince}

Afin de définir le célibataire de la littérature fantastique fin-de-siècle, Prince s'attarde d'abord à la société qui l'a vu naître, plus particulièrement aux changements de mentalité sociale. À cette époque, être célibataire semble devenir une mode : une élite de célibataires dirige la France (Thiers, Gambetta, etc.), les réunions entre hommes se multiplient et l'individualisme triomphe. Néanmoins, le célibataire est aussi perçu comme le signe d'une décadence sociale, ce qui lui octroie une double posture idéologique : autant il captive par son choix délibéré de vivre seul et libre de toute responsabilité morale, sociale, familiale et financière, autant il inquiète par son refus du devoir social qu'est la reproduction de l'espèce. Apparaissant comme une résistance consciente 
(Célibataires, 24), le célibataire est considéré par la bourgeoisie comme une anormalité, un monstre social qui charme et effraie par son caractère énigmatique. Ainsi, à la fois effet de réel et effet fantastique, le célibataire semble à lui seul constituer « une intrusion brutale du mystère dans le cadre de la vie réelle»(Castex 8) par la menace qu'il représente pour le couple, la famille, la société et les mœurs.

Pour sa définition du célibataire, Prince énonce quatre éléments constitutifs : s'agissant, «régulièrement, [d']un personnage presque toujours identique - masculin, solitaire, souvent reclus [et] souvent cultivé »(Célibataires, 12), le célibataire est à la fois misanthrope, misogyne, misogame et, souvent, monomane (le tout fréquemment accompagné d'une crise identitaire). Plus encore, Prince souligne que le rôle du célibataire peut varier au sein du récit; en effet, «[q]u'il s'agisse du personnage principal, du narrateur, de la victime, voire même de la créature fantastique, la plupart des actants [dans les récits fantastiques] sont célibataires » (12-13). Enfin, elle souligne que le célibataire - bien nanti et titré - n'est ni un père, ni un homme comme les autres, il est un grand défaitiste de l'amour normal, d'où sa recherche constante d'idéal, ce qui l'amène sur la voie de l'excentricité et de la perversion. Mais surtout, elle affirme que le célibataire n'est pas une femme. Ce faisant, elle fait siens les nombreux préjugés misogynes fin-de-siècle pour l'exclure de son propos : «Non mariée, la femme est fille ou 'reste fille' : c'est-à-dire rien » (13). Plus encore, Prince ajoute que :

La femme, réputée naturellement apeurée, craintive, stupide et coquette ne peut tendre à la même crédibilité que le célibataire, et elle ne saurait avoir les mêmes réflexes face à un événement étrange qu'un homme réfléchi, à l'esprit rassis, entièrement disponible pour analyser le phénomène qu'il a sous les yeux : «il faut qu'il s'agisse d'un homme» [...]. Une femme racontant qu'elle a vu un fantôme a-t-elle la moindre chance d'être prise au sérieux ? (13-14)

Mais que faire d'une écrivaine telle que Rachilde et de ses personnages de femmes répondant, en grande partie, aux critères de Prince ? À mon sens, la femme célibataire des romans de Rachilde présente une parenté - notable et signifiante - avec le célibataire des récits fantastiques fin-de-siècle. Ils constituent, dans les deux cas, une menace par rapport à l'ordre établi ; tout comme le célibataire, son homologue féminin rachildien renie son rôle vital de reproductrice. Elle incarne également cet «être de la négation et de l'anormalité en tant que nuisance, inutilité et impossibilité sociale »(30). 
Toutefois, étant donné la différence des conditions d'existence de l'homme et de la femme à la fin du dix-neuvième siècle, les conditions du célibat de l'un et de l'autre diffèrent également. Effectivement, à cette époque, le droit à la liberté ou au célibat n'était pas octroyé aux femmes; celles-ci n'étaient donc jamais totalement et véritablement libres. Pour parvenir à une même aisance financière et matérielle, voire à une certaine indépendance, que le célibataire, possédant fortune et titre, la femme doit parfois emprunter d'autres avenues. Par exemple, elle peut refuser de se remarier lorsqu'elle devient veuve (et héritière); elle peut être entretenue - plus ou moins en continu - par un homme dans une relation hors mariage (conservant ainsi une certaine liberté) et il y a, bien évidemment, celle qui ne s'est jamais mariée, la vieille fille ; toutefois pour obtenir indépendance et liberté, il faut qu'elle soit, au préalable, nécessairement titrée. Mais peu importe sa situation, on retrouvera chez cette femme célibataire « un mépris, voire un rejet radical de l'amour [normal, socialement parlant,] et de son institution bourgeoise, le mariage » (50-51). Les deux exemples qui suivent permettront de montrer la pertinence de l'étude du personnage de femme célibataire chez Rachilde.

\section{La célibataire prêtresse}

Dans La Jongleuse (1900), Rachilde met en scène Éliante Donalger, une créole française âgée de trente-cinq ans, rencontrant Léon Reille, un jeune étudiant en médecine de vingt-deux ans captivé par elle et souhaitant ardemment devenir son amant, et même son époux. Mariée à dix-sept ans, veuve à trente ans (79) d'un officier de la marine d'une quarantaine d'années défiguré par la guerre, Éliante choisit délibérément de ne pas se remarier et embrasse son état de femme célibataire et libre :

[...] je ne veux point me remarier, mon cher enfant, j'ai passé l'âge... il faut que je demeure libre. Je tiens à courir aux heures que je choisis, sortir seule, fuir souvent l'endroit que j'habite, parce que je suis un peu sauvage, il faut que j'aille à l'aventure selon mon caprice d'ancienne bête élevée à quatre pattes.

$[\ldots]$ je ne veux plus d'un mari, parce que c'est trop lourd, et je ne veux pas d'un amant parce que... J'ai charge d'âme ici. (79)

Riche héritière et sans enfant, Éliante recueille chez elle la famille très pauvre de son défunt époux, puisqu'elle lui doit toute sa fortune (74). À plusieurs reprises, elle 
mentionne à Léon ses pénibles devoirs envers son beau-frère, qu'elle doit ultimement enterrer, et sa jeune nièce Missie, qu'elle doit marier (101). Perçu comme une prison, le mariage la brime, l'empêche d'exister tout simplement. Il en va de même pour la famille qui constitue, pour Éliante, un demi-deuil quotidien (74) et ses «derniers liens sociaux » (170), dont elle se souhaite se libérer.

Esprit libre difficilement fait pour vivre en société, Éliante apparaît comme un être à part, unique, et fortement décalé et dépareillé par rapport aux autres personnages du récit. En effet, elle est un véritable objet de curiosité et d'étrangeté pour ceux qui la côtoient : «Ni mon oncle, ni moi, ni personne nous ne saurons jamais ce qu'elle est au juste. Elle sort quand elle veut, fait tout ce qu'il lui plaît et ne nous consulte pas. Elle reste fermée comme sa chambre »(154). La façon dont elle est décrite, l'emprise physique et psychologique qu'elle a sur Léon, et sa conception de l'amour et de la volupté participent également à faire d'elle une créature foncièrement fantastique : «Elle semblait à la fois très chez elle et très en dehors de tous les mondes » (37); « cette femme mystérieuse qui semblait [...] arriver de plus loin que... la terre ferme » (202). Léon note ses traits de poupée artificielle (26), ses mouvements de chat (28), ses yeux phosphorescents (190) et semble à chaque fois affecté (parfois même effrayé) par Éliante lorsqu'il est en sa présence : on parle de vertige, de frayeur, d'état d'hypnose, de tremblement de rage, d'impressions de perdre la tête, etc. Et ces effets, presque hallucinatoires, sont voulus de la part d'Éliante : «Je veux passer sur ta vie comme le rêve et non comme une réalisation vulgaire. Je sais que je suis l'unique... » (149) ; «Car, moi, je garde tout, j'emporte tout... Je suis votre rêve...» (168). De même, le titre du roman, La Jongleuse, fait référence à Éliante qui, malgré son statut social élevé, devient une saltimbanque jongleuse de couteaux le temps de divertir ses invités lors de ses soirées. Masquée et à demi nue, elle termine son numéro en rattrapant l'ultime couteau entre ses seins (un fourreau y est dissimulé à cet effet), faisant alors croire à l'assistance qu'elle en meurt, ce qui sera effectivement le cas à la toute fin du roman (Éliante commet ce geste de façon délibérée). Ce talent, ou don, renforce sa non-appartenance à son entourage et l'isole d'autant plus : «[Éliante était] debout et jongla[it], séparée de sa famille, de la société, du monde entier, de toute l'humanité par l'énigme de sa comédie 
perpétuelle »(143). Ainsi, Éliante remplit bien son rôle de célibataire excentrique et solitaire.

La question du double, thème éminemment fantastique, est aussi présente dans le roman de Rachilde et vient accentuer l'étrangeté fantastique du personnage d'Éliante. Enfermé avec cette dernière dans sa chambre, qui est comparée à la fois à un «temple » (104) et à «une caverne où [l'on] étouffe » (106), Léon contemple une multitude de petites idoles que son défunt mari rapportait de ses voyages en Chine. Le jeune homme est à la fois horrifié et fasciné par ces petits objets, car ils sont, en fait, tous à l'effigie d'Éliante (120) : «elles me ressemblent parce que c'est moi qu'elles représentent » (120). En effet, non seulement Henri Donalger vouait, de son vivant, un culte à sa femme, mais elle aussi se voit en tant qu'idole, prêtresse de l'Amour, libre de toute attache terrestre et en connexion directe avec son dieu : « il y a une fournaise en moi. Je suis habitée par un dieu » (150) ; «Je suis une recluse libre, une sorte de religieuse émancipée, une prêtresse laïque. Je ne veux que vous convertir à ma religion, qui est l'unique » (84). Avec Éliante « s'exaltant à toucher des idoles » (115) à son image, il s'agit bien là d'un culte, d'un amour foncièrement narcissique, typique du personnage célibataire fin-de-siècle. Ce que refuse catégoriquement Éliante, c'est « la passion ordinaire » (84), où l'amour physique, ou rapport sexuel, que ce soit avec Léon ou quiconque, constitue une nuisance dans l'atteinte du divin et du plaisir, ce qui la mène vers des amours nécessairement infécondes, donc socialement inutiles. À juste titre, la relation entre Éliante et Léon est surtout celle d'une maîtresse et de son élève ayant pour but d'enseigner un certain Amour et «[d']inspirer le goût du beau » (167). L'amour que prône Éliante est d'un tout autre type, davantage spirituel et divin, où la Beauté et la Volupté en forment le cœur :

Je suis réellement amoureuse de tout ce qui est beau, bon, me paraît un absolu, la définition même de la volupté. Mais ce n'est pas un but, le plaisir ; c'est une manière d'être. Moi, je suis toujours... heureuse. [...] je n'ai pas besoin de la caresse humaine pour arriver au spasme... Il me suffit d'être... [...] car je porte en moi le secret de toutes les sciences en ne sachant qu'aimer. J'ai le dégoût de l'union, qui détruit ma force, je n'y découvre aucune plénitude voluptueuse. Pour que ma chair s'émeuve et conçoive l'infini du plaisir, je n'ai pas besoin de chercher un sexe à l'objet de mon amour ! (49-50) 
Être de plaisir et grande amoureuse, elle met en pratique ce qu'elle prêche en montrant à Léon sa propre façon d'atteindre la jouissance. Recluse dans l'intimité d'une « petite chapelle trop moderne » (48) construite en l'honneur d'un énorme vase oriental et ancien d'une beauté exquise et suprême, Éliante pratique l'auto-érotisme : « La jeune femme, l'œil mi-clos, s'attacha davantage au col de l'amphore. Elle pressa ses deux bras autour du bourrelet de cette chair de pierre, s'inclina sur l'ouverture en corolle, baisant le vide » (49); «Elle ne s'offrait pas à l'homme; elle se donnait au vase d'albâtre, le personnage insensible de la pièce » $(50)$; « et elle eut un petit râle de joie imperceptible, le souffle même du spasme » (51). Par cette pratique sexuelle, jugée «scandaleu[se et] abominable » (51) par Léon qui en est témoin, ainsi que par sa doctrine d'un amour autre que celui prôné par la société, le personnage d'Éliante s'inscrit bel et bien dans le sillage du célibataire fantastique fin-de-siècle. Enfin, on trouve chez Éliante un discours dénonçant la situation matrimoniale des femmes et teinté de misogynie, ce qui n'est pas sans rappeler la mentalité fin-de-siècle :

[...] la femme honnête est celle qui cède. Vous avez tous [en parlant des hommes] inventé cela dès votre berceau pour la plus grande commodité de vos futures alcôves, et vous l'avez tellement répété que les plus niaises le croient, aujourd'hui [...]. Elles naissent aussi en le croyant, et on entend même les jeunes filles charmantes déclarer, les poings en l'air, qu'elles céderont, l'occasion offerte, pour s'assurer des droits qui ne s'acquièrent qu'avec une expérience diabolique. (171-172)

Voulant être chérie, adulée, mais « heureuse toute seule, les bras bien croisés sur [s]a poitrine, les cuisses jointes hermétiquement, avec le sourire des vierges qui communient» (167), l'Éliante de Rachilde constitue une femme célibataire forte, spirituelle, engagée et certaine de ses convictions extra-terrestres; elle est, de toute évidence, caractérisée par cette résistance consciente que Prince reconnaît aux célibataires masculins, qui refusent de se prêter au jeu banal de la société.

\section{La célibataire animale}

Pour ce qui est du célibat de Laure Lordès, héroïne de L'Animale (1893), il en va tout autrement. Tout d'abord, il est question, dans le roman, des origines du personnage, de sa jeunesse, menant ensuite à sa vie d'adulte à Paris. Cette genèse montre la nature intrinsèque, voire la prédisposition héréditaire (à la façon naturaliste), de Laure, qu'on 
nous dit «pourrie » (31), et ce, même avant sa naissance. En effet, les Lordès, ayant épuisé toutes les ressources connues pour avoir un enfant, ont eu recours «aux magies des aliments excitants pour créer » (33) (concoctions d'herbes et d'épices exotiques, plats aphrodisiaques, « liqueurs sorcières » (33), etc.). Par conséquent, Laure, «issu[e] de leurs petites infamies » et de «leurs péchés » (31-33), naît «avec le germe du mal » (31) et le « signe fatal de la luxure » (101), ce qui, d'emblée, fait d'elle un être à part. Durant son enfance et sa jeunesse passées à Estérac, elle s'amuse à dévergonder les garçons du voisinage et s'initie à la sexualité dès l'âge de treize ans. Son secret, bien gardé jusqu'à l'aube de ses vingt-un ans, finit par s'ébruiter ; Laure est alors rejetée à la fois par Henri, qui rompt les fiançailles et quitte Estérac pour Paris, et par ses parents qui la chassent brutalement. Laure, soulagée d'avoir évité le mariage, décide de retrouver son ex-fiancé à Paris : «Tant mieux, je préfère ça, je serai votre maîtresse au lieu d'être votre femme, voilà tout...» (123) ; «Je n'ai pas besoin de vous pour me cacher. J'aurais pu vivre ailleurs... » (141)

Il est à noter que, tout au long du roman, Laure n'a, à aucun moment, honte d'elle-même ou n'émet un quelconque regret par rapport à ses actes ; elle est un esprit libre de toute convention sociale, un esprit «sauvage» (174), «animal» (37), «primitif» (173). Le scandale lui a servi à se sortir d'une situation non voulue : «Se marier? Elle n'y tenait pas beaucoup, ayant bien deviné que le mariage n'assouvissait pas les créatures de sa trempe. Elle rêvait d'une autre vie, d'un cloître, si on voulait, mais d'un cloître où l'on se trouverait deux de sexe différent, perpétuellement en tête-à-tête sur des coussins de velours » (62). Malheureusement pour elle, Henri ne sera pas cet autre sexe qui satisfera tous ses désirs. Ce dernier lui loue à Paris un «modeste appartement à un sixième étage », un endroit d'amour (141), où Laure et lui se croient heureux pendant toute une année (141). Une nuit, la jeune femme s'aventure sur le toit et assiste alors à « une ronde infernale » de chats qui tentent de la séduire, la croyant l'une des leurs avec sa longue tresse de cheveux noirs «qu'il[s] prenai[ent] de plus en plus pour une fourrure » (25). C'est à partir de ce moment que la métamorphose de Laure s'entame véritablement. Dès qu'elle entre en contact avec ces animaux, elle prend conscience qu'elle n'est «pas une femme comme les autres » et «se découvr[e] une spéciale nature de brute, des entrailles de bête correspondantes aux instincts délicieux du petit chat, car 
les chats sont, dit-on, tout simplement des esprits dévoyés qui rôdent, vêtus de fourrure, pour s'efforcer de reconquérir leur ancien corps féminin »(151). Peu à peu, tous les repères sociaux de Laure sont abandonnés : elle ne parle à personne, elle ne sort pratiquement plus de chez elle et, lorsqu'il y a rupture définitive avec Henri, sa réclusion devient, pour ainsi dire, totale ; son unique contact avec un être vivant est son chat, Lion. De ce fait, le personnage de Laure est l'exemple parfait de la femme célibataire solitaire et misanthrope, parce que faisant davantage partie du monde animal que celui des humains : «Non, les hommes ne sont pas dignes de mes ardeurs, et je ne veux plus m'égarer encore à fouiller ce cloaque. Je suis lasse... Tant pis ! Que cela finisse comme ça voudra » (195). Et cette fin « comme ça voudra » va mener Laure à la folie et à une relation amoureuse avec son chat. Sans cesse assoiffée d'amour et de luxure et en recherche constante de l'amour idéal et à sa mesure (tel le célibataire fin-de-siècle), Laure, désespérée, se laisse corrompre, et même asservir, par Lion, seul véritable mâle dominant du roman : «Un chat, un chien, un homme, un monstre, pourvu qu'elle fût adorée au moment où elle désirerait qu'on l'adorât !... »(223). Plus encore, l'extrait suivant montre bien l'état de dégradation et de folie dans lequel sombre Laure, dont l'identité glisse peu à peu vers une réelle animalité :

Dans l'étroitesse de leur existence, où l'amour d'un homme ne trouvait plus sa place, elle fit ses délices de son chat et jouit véritablement d'un bonheur animal très exquis. Ces deux simples créatures, si naturellement compliquées, s'entendaient à merveille, et ressentaient les mêmes ennuis, les mêmes impatiences, les mêmes joies. (234-235)

Toute détraquée par le contact de cette fourrure, qu'elle galvanisait de sa chaude humanité, où elle introduisait son fluide cérébral, elle perdait, abîmait son esprit dans la contemplation de l'impossible. Elle en avait aussi la frayeur et l'attirance, comme si, par ces petites fentes lumineuses où brillaient les étincelles d'un feu diabolique, un vide l'aspirait toute [...]. Quelqu'un, quelque chose, peut-être l'âme de la bête elle-même [...] lui lançait un sort derrière cet ondoyant fantôme de chat, l'envoûtait, et elle se laissait docilement subjuguer, satisfaite de perdre en intelligence ce que Lion lui rendait en caresses. (237-238)

Dès lors, un point de non-retour semble avoir été définitivement franchi : «Une fois, [...] elle osa jouer avec la mignonne corne de corail s'érigeant parmi les soies rousses de son ventre, et eut le geste moqueur de la tourner contre leur infortune, la 
jettatura!» (239) À la suite de cet événement, Lion s'enfuit un certain temps, pendant lequel Laure finit par rencontrer l'amant idéal tant espéré. Afin de lui prouver sa complète dévotion, elle se laisse emprisonner chez elle par cet homme, dont elle ignore le nom. Séquestrée, Laure retrouve Lion «cria[nt] de rage », «méconnaissable, la fourrure souillée, les prunelles sanglantes, la gueule baveuse, si maigre qu'il était comme grandi par la sveltesse fantastique de son corps » (264). Au sein du roman, non seulement Laure est-elle présentée en tant que créature fantastique par rapport à son entourage (on note à la fois son physique de félin et ses mœurs amoureuses étranges), mais aussi en tant que victime du fantastique par son chat Lion, qui est devenu - à la fin du récit - ce véritable monstre l'entraînant dans un ultime «accouplement bestial » qui scelle sa propre métamorphose en bête/animale :

Lion avait vraiment l'air de se venger [...]. Il lui fallait tout détruire, tout profaner [...]. Il lui fendit la lèvre inférieure, [...] cracha sa bave empoissonnée [...] entre ses dents blanches [...]. Une bête, le museau rongé au ras des dents, le nez coupé, [...] une bête sans paupières, [...] une bête aux mamelles pendantes et fendues [...] qui se terminait en queue jaune annelée de velours. À travers son voile de sang, Laure s'était vue dans la glace. (266-268)

Bien entendu, «la femme métamorphosée en bête » (268-269) qu'est devenue Laure ne peut exister (même chose pour Lion) ; ils ne font désormais plus partie de ce monde, d'où leur chute vers la mort.

Enfin, contrairement à Éliante - chez qui se rejoigne une multitude de discours sur l'Amour, la religion, le plaisir, bref, où il y a une réflexion aboutie -, Laure est une célibataire animale, une sorte de résistance inconsciente tout en pulsions et en instincts, une force sur-naturelle bouleversant, voire profanant, tout sur son passage (famille, mœurs, religieuses et sociales, etc.), ce qui renforce son exclusion de la société. Sa réclusion, qui en est le résultat, donne lieu à sa monomanie grandissante et à ses amours ambigües (alors qu'elle est seule avec Lion) ainsi qu'à sa transformation finale en animale. Dans ce roman, Rachilde a donc mis en scène la genèse, la création, d'une telle femme célibataire fantastique, qui, dès sa naissance, présente des anormalités ${ }^{1}$, des « anomalies » (30), prédisant qu'elle sera non seulement incapable de vivre en société, mais qu'elle constituera aussi une menace de taille pour celle-ci, puisqu'elle appartient davantage à des temps anciens et primitifs. 


\section{Conclusion}

Pour les besoins de cet article, il a fallu se restreindre en ne présentant que deux exemples de personnages de femmes célibataires rachildiennes. Il va de soi qu'il en existe d'autres, par exemple celui de la célibataire déchue, Marie Faneau, dans Le Grand Saigneur (1922). Artiste-peintre, vivant de son art et faisant même vivre son frère, un noceur incorrigible, Marie perd non seulement sa liberté et son indépendance, en épousant - presque contre son gré - le marquis de Pontcroix, soupçonné d'être un vampire, mais aussi son identité. Lorsqu'il y a union, chez Rachilde, une crise identitaire, voire une aliénation, en découle. Force est de constater que l'écrivaine décadente a bel et bien adapté, et même féminisé, le personnage du célibataire fantastique fin-de-siècle, en lui attribuant les mêmes ressorts fantastiques ; simple objet dans les textes de l'époque, le personnage féminin devient un véritable sujet sous la plume de Rachilde. Figure importante, voire centrale, de la littérature fantastique fin-de-siècle, la femme constitue « une intrusion brutale du mystère dans la vie réelle »(Castex 8). La représenter seule, isolée de la société, indépendante, libre et douée d'un appétit sexuel jugé étrange, c'est subvertir son rôle social, briser les carcans, annonçant ainsi une rupture identitaire, chère à cette littérature fantastique fin-de-siècle.

\section{Bibliographie}

Castex, Pierre-Georges. Le Conte fantastique en France de Nodier à Maupassant. Paris : Corti, 1951.

Prince, Nathalie. Le Fantastique, Paris : Colin, 2008 (coll. «128»).

---. Les Célibataires du fantastique: Essai sur le personnage célibataire dans la littérature de la fin du XIX $X^{e}$ siècle. Paris : L'Harmattan, 2002.

Rachilde. L'Animale. Paris : Mercure de France, 1993 [1893].

---. La Jongleuse. Paris : Des femmes, 1982 [1900].

\section{Note}

1. On dit même de Laure « [qu'] elle est marquée d'une plaie hideuse, [qu'] elle tarée du signe fatal de la luxure » (101). 